ESAIM: PROCEEDINGS, October 2007, Vol. 22, 21-39

Gabriel Caloz \& Monique Dauge, Editors

\title{
CONTROLLABILITY AND NONLINEARITY
}

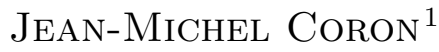

\begin{abstract}
We present here three methods (return method, quasi-static deformations, power series expansions) to get controllability results for control systems modeled by partial differential equations when the linearized control system around the equilibrium is not controllable.
\end{abstract}

\section{INTRODUCTION}

When one wants to study the controllability of a control system around an equilibrium, the first thing to do is to linearize the control system around this equilibrium. If the linearized control system is controllable, one deduces from the inverse function theorem that the nonlinear control system is locally controllable around this equilibrium, at least in finite dimension. In infinite dimension, this remains essentially true. However, for interesting control systems, some loss of derivatives may appear: See in particular the papers [1-3] by Karine Beauchard, as well as our joint work [4] with Karine Beauchard.

In this talk, we consider the case where the linearized control system around the considered equilibrium is not controllable.

In finite dimension the usual methods used to treat this case rely on iterated Lie brackets: see Section 2 . In infinite dimensions, iterated Lie brackets are not well defined for many interesting cases (see Section 3 for an example). We present here three methods to get controllability results for some control systems modeled by partial differential equations even if the linearized control system around the equilibrium is not controllable. These methods are

(1) The return method,

(2) Quasi-static deformations,

(3) Power series expansion.

For simplicity the presentation of these three methods will be made essentially in finite dimension, even if this is for infinite-dimensional control systems that there are interesting.

\section{ItERATED LIE BRACKETS AND CONTROLLABILITY IN FINITE DIMENSION}

Let us consider the following control system

$$
\dot{x}=f(x, u):=f_{0}(x)+\sum_{i=1}^{m} u_{i} f_{i}(x)
$$

\footnotetext{
1 Université de Paris-Sud and Institut universitaire de France, Département de Mathématique, 91405 Orsay, France, JeanMichel.Coron@math.u-psud.fr

(C) EDP Sciences, SMAI 2007
} 
where the state is $x \in \mathbb{R}^{n}$ and the control is $u:=\left(u_{1}, \ldots, u_{m}\right)^{\operatorname{tr}} \in \mathbb{R}^{m}$. (Here and in the following, vectors of $\mathbb{R}^{k}$ are column vectors and $M^{\text {tr }}$ denotes the transpose of the matrix $\left.M\right)$. The maps $f_{i}, i=1, \ldots, m$, are assumed to be of class $C^{\infty}$ from $\mathbb{R}^{n}$ into $\mathbb{R}^{n}$. We assume that

$$
f_{0}(0)=0 .
$$

We use the following definition of small-time local controllability.

Definition 1. The control system (1) is small-time locally controllable at $(0,0) \in \mathbb{R}^{n} \times \mathbb{R}^{m}$ if, for every $\varepsilon>0$, there exists $\eta>0$ such that, for every $a \in \mathbb{R}^{n}$ and for every $b \in \mathbb{R}^{n}$ such that $|a|+|b|<\eta$, there exists $u \in L^{\infty}\left((0, \varepsilon) ; \mathbb{R}^{m}\right)$ such that

$$
\begin{gathered}
|u(t)|<\varepsilon, t \in(0, \varepsilon), \\
(\dot{x}=f(x, u(t)) \text { and } x(0)=a) \Rightarrow(x(\varepsilon)=b) .
\end{gathered}
$$

In order to see if the control system (1) is small-time locally controllable at $(0,0) \in \mathbb{R}^{n} \times \mathbb{R}^{m}$, the first thing to do is to look at the controllability of the linearized control of $\dot{x}=f(x, u(t))$ at $(0,0)$, i.e., the linear control system

$$
\dot{x}=\frac{\partial f}{\partial x}(0,0) x+\frac{\partial f}{\partial u}(0,0) u,
$$

where the state is $x \in \mathbb{R}^{n}$ and the control is $u \in \mathbb{R}^{m}$. One has the following classical result.

Theorem 2. If the linear control system (5) is controllable, then the nonlinear control system (1) is small-time locally controllable around $(0,0) \in \mathbb{R}^{n} \times \mathbb{R}^{m}$.

Of course "the linear control system (5) is controllable" just means that, for every $\varepsilon>0$, for every $a \in \mathbb{R}^{n}$ and for every $b \in \mathbb{R}^{n}$, there exists $u \in L^{\infty}\left((0, \varepsilon) ; \mathbb{R}^{m}\right)$ such that

$$
(\dot{x}=A x+B u(t) \text { and } x(0)=a) \Rightarrow(x(\varepsilon)=b) .
$$

This simple but powerful theorem is quite interesting since one has the following theorem (Kalman's rank condition).

Theorem 3. The linear system $\dot{x}=A x+B u$, where the state is $x \in \mathbb{R}^{n}$ and the control is $u \in \mathbb{R}^{m}$, is controllable if and only if the vector subspace of $\mathbb{R}^{n}$ spanned by the set of $A^{i} B u, u \in \mathbb{R}^{m}, i \in\{0, \ldots, n-1\}$, is equal to $\mathbb{R}^{n}$.

The difficulties start when the linearized control system (5) is not controllable. In this case, one can deduce nothing about the small-time local controllability of $(1)$ around $(0,0) \in \mathbb{R}^{n} \times \mathbb{R}^{m}$. However, one has a useful tool to treat this case, namely iterated Lie brackets. Let us start by recalling some classical definitions.

Definition 4. Let

$$
\begin{aligned}
& X:=\left(X^{1}, \ldots, X^{n}\right)^{\operatorname{tr}} \in C^{1}\left(\mathbb{R}^{n} ; \mathbb{R}^{n}\right), \\
& Y:=\left(Y^{1}, \ldots, Y^{n}\right)^{\operatorname{tr}} \in C^{1}\left(\mathbb{R}^{n} ; \mathbb{R}^{n}\right) .
\end{aligned}
$$

The Lie bracket $[X, Y]:=\left([X, Y]^{1}, \ldots,[X, Y]^{n}\right)$ of $X$ and $Y$ is the element of $C^{0}\left(\mathbb{R}^{n} ; \mathbb{R}^{n}\right)$ defined by

$$
[X, Y]^{j}(x):=\sum_{k=1}^{n} X^{k}(x) \frac{\partial Y^{j}}{\partial x_{k}}(x)-Y^{k}(x) \frac{\partial X^{j}}{\partial x_{k}}(x),
$$

for every $j \in\{1, \cdots, n\}$ and for every $x \in \mathbb{R}^{n}$. 
Definition 5. Let

$$
X \in C^{\infty}\left(\mathbb{R}^{n} ; \mathbb{R}^{n}\right) \text { and } Y \in C^{\infty}\left(\mathbb{R}^{n} ; \mathbb{R}^{n}\right) .
$$

One define, by induction on $k \in \mathbb{N}$, ad $d_{X}^{k} Y \in C^{\infty}\left(\mathbb{R}^{n} ; \mathbb{R}^{n}\right)$ by

$$
\begin{gathered}
a d_{X}^{0} Y=Y, \\
a d_{X}^{k+1} Y=\left[X, a d_{X}^{k} Y\right], \forall k \in \mathbb{N} .
\end{gathered}
$$

The pioneer work showing the interest of iterated Lie brackets for the controllability problem are due to Robert Hermann [24], Tadashi Nagano [28] and Claude Lobry [27].

Let us just explain why it is natural to consider Lie brackets to study the controllability. Let us first assume that our control system (1) is a driftless control system and that $m=2$. In other words $f_{0}=0$ and our control system (1) is

$$
\dot{x}=u_{1} f_{1}(x)+u_{2} f_{2}(x) .
$$

Let us start from $0 \in \mathbb{R}^{n}$. One knows how to move in the (oriented) directions $\pm f_{1}(0)$, even with small controls: it suffices to take $u_{1}=\eta \neq 0$ and $u_{2}=0$. Similarly, one knows how to move in the directions $\pm f_{2}(0)$. Let us explain how one can move in the directions $\pm\left[f_{1}, f_{2}\right](0)$. Let $\Psi_{i}: \mathbb{R} \times \mathbb{R}^{n} \rightarrow \mathbb{R},(t, x) \rightarrow \Psi_{i}^{t}(x)$ be the flow associated to $f_{i}, i \in\{1,2\}$. In other words,

$$
\frac{\partial \Psi_{i}}{\partial t}=f_{i}\left(\Psi_{i}\right), \Psi_{i}^{0}(x)=x
$$

Let us fix two real numbers $\eta_{1} \in \mathbb{R}$ and $\eta_{2} \in \mathbb{R}$. Elementary computations lead to

$$
\lim _{\varepsilon \rightarrow 0} \frac{\left(\Psi_{2}^{-\eta_{2} \varepsilon}\left(\Psi_{1}^{-\eta_{1} \varepsilon}\left(\Psi_{2}^{\eta_{2} \varepsilon}\left(\Psi_{1}^{\eta_{1} \varepsilon}(a)\right)\right)\right)\right)}{\varepsilon^{2}}=\eta_{1} \eta_{2}\left[f_{1}, f_{2}\right](a) .
$$

We start from $0 \in \mathbb{R}^{n}: x(0)=0$. For the control, we choose the following functions:

- $\left(u_{1}(t), u_{2}(t)\right)=\left(\eta_{1}, 0\right)$, for $t \in(0, \varepsilon)$,

- $\left(u_{1}(t), u_{2}(t)\right)=\left(0, \eta_{2}\right)$, for $t \in(\varepsilon, 2 \varepsilon)$,

- $\left(u_{1}(t), u_{2}(t)\right)=\left(-\eta_{1}, 0\right)$, for $t \in(2 \varepsilon, 3 \varepsilon)$,

- $\left(u_{1}(t), u_{2}(t)\right)=\left(0,-\eta_{2}\right)$, for $t \in(3 \varepsilon, 4 \varepsilon)$,

Then, as $\varepsilon \rightarrow 0$,

$$
x(4 \varepsilon)=\eta_{1} \eta_{2} \varepsilon^{2}\left[f_{1}, f_{2}\right](0)+o\left(\varepsilon^{2}\right) .
$$

Therefore we are able to move in the directions $\pm\left[f_{1}, f_{2}\right](0)$.

We can keep going and considered iterated Lie brackets: one has the following theorem, due to Petr Rashevski [30] and Wei-Liang Chow [6]

Theorem 6. Let Lie $\left\{f_{1}, f_{2}, \ldots, f_{m}\right\}$ be the Lie algebra generated by the $f_{1}, f_{2}, \ldots, f_{m}$, i.e., the smallest of the vector subspaces $E$ of $C^{\infty}\left(\mathbb{R}^{n}, \mathbb{R}^{n}\right)$ satisfying the following two properties:

$$
\begin{aligned}
& {[X, Y] \in E, \forall(X, Y) \in E^{2},} \\
& f_{i} \in E, \forall i \in\{1,2, \ldots, m\} .
\end{aligned}
$$

Assume that

$$
\left\{h(0) ; h \in \operatorname{Lie}\left\{f_{1}, f_{2}, \ldots, f_{m}\right\}\right\}=\mathbb{R}^{n} .
$$


Then the control system

$$
\dot{x}=\sum_{i=1}^{m} u_{i} f_{i}(x)
$$

is small-time controllable around $(0,0) \in \mathbb{R}^{n} \times \mathbb{R}^{m}$.

Example 7. (Nonholonomic integrator.) We take $n=3, m=2$, and, for every $x=\left(x_{1}, x_{2}, x_{3}\right)^{\operatorname{tr}} \in \mathbb{R}^{3}$,

$$
\begin{gathered}
f_{1}(x)=\left(1,0,-x_{2}\right)^{\mathrm{tr}}, \\
f_{2}(x)=\left(0,1, x_{1}\right)^{\mathrm{tr}} .
\end{gathered}
$$

Hence our control system is

$$
\dot{x}_{1}=u_{1}, \dot{x}_{2}=u_{2}, \dot{x}_{3}=x_{1} u_{2}-x_{2} u_{1},
$$

The linearized control system around $(0,0) \in \mathbb{R}^{3} \times \mathbb{R}^{2}$ is

$$
\dot{x}_{1}=u_{1}, \dot{x}_{2}=u_{2}, \dot{x}_{3}=0,
$$

which is not controllable (one cannot change $x_{3}$ ). However

$$
\left[f_{1}, f_{2}\right](x)=(0,0,2)^{\operatorname{tr}}, \forall x \in \mathbb{R}^{3} .
$$

Hence, $f_{1}(0), f_{2}(0)$ and $\left[f_{1}, f_{2}\right](0)$ span $\mathbb{R}^{3}$ and Theorem 6 gives us that the control system (13) is small-time locally controllable around $(0,0) \in \mathbb{R}^{3} \times \mathbb{R}^{2}$.

Even if there is a drift term (i.e. $f_{0} \neq 0$ ), the Lie brackets are useful. Indeed, let us for example consider the case where $m=1$ and

$$
f(x, u)=f_{0}(x)+u f_{1}(x) .
$$

with, again, (2). We still start from $0 \in \mathbb{R}^{n}$. Of course one knows how to move in the directions $\pm f_{1}(0)$. Let us explain how to move in the direction $\pm\left[f_{1}, f_{0}\right](0)$. Let $\eta \in \mathbb{R}$. For $\varepsilon>0$, consider the control $u:[0,2 \varepsilon] \rightarrow \mathbb{R}$ defined by

$$
\begin{aligned}
& u(t)=-\eta \text { for } t \in(0, \varepsilon), \\
& u(t)=\eta \text { for } t \in(\varepsilon, 2 \varepsilon) .
\end{aligned}
$$

Let $x:[0,2 \varepsilon] \rightarrow \mathbb{R}^{n}$ be the solution of the Cauchy problem

$$
\dot{x}=f_{0}(x)+u(t) f_{1}(x), x(0)=0 .
$$

Then, simple computations show that, as $\varepsilon \rightarrow 0$,

$$
x(2 \varepsilon)=\varepsilon^{2} \eta\left[f_{0}, f_{1}\right](0)+o\left(\varepsilon^{2}\right) .
$$

Hence we have succeeded to move in the directions $\pm\left[f_{0}, f_{1}\right](0)$. Unfortunately the case of systems with a drift term is more complicated that the case of driftless control systems: the iterated Lie brackets are not all "good". For example, consider the following control system 


$$
\dot{x}_{1}=x_{2}^{2}, \dot{x}_{2}=u
$$

where the state is $x=\left(x_{1}, x_{2}\right)^{\operatorname{tr}} \in \mathbb{R}^{2}$ and the control is $u \in \mathbb{R}$. Hence

$$
f_{0}(x)=\left(x_{2}^{2}, 0\right)^{\operatorname{tr}}, f_{1}(x)=(0,1)^{\operatorname{tr}} .
$$

One has

$$
\left[\left[f_{1}, f_{0}\right], f_{1}\right](x)=-(2,0)^{\mathrm{tr}} .
$$

However, for a trajectory $t \rightarrow(x(t), u(t))$ of $(17), t \rightarrow x_{1}(t)$ is nondecreasing. Hence one cannot move in the direction $\left[\left[f_{1}, f_{0}\right], f_{1}\right](0)$. For necessary conditions and sufficient conditions for small-time local controllability, see, e.g., [12, Chapter 3] and the references therein. Let us however point out that one does not know any (effective) necessary and sufficient condition for small-time local controllability.

\section{ITERATED LIE BRACKETS AND CONTROL SYSTEMS IN INFINITE DIMENSIONS}

In this section, we explain why iterated Lie brackets are less useful in infinite dimensions. Let $L>0$. Let us consider the following control system, which is probably the simplest control system in infinite dimensions.

$$
\begin{gathered}
y_{t}+y_{x}=0, t \in[0, T], x \in[0, L], \\
y(t, 0)=u(t), t \in[0, T] .
\end{gathered}
$$

Here, $T>0$ and $L>0$ are given and, at time $t \in[0, T]$, the state is $y(t, \cdot):(0, L) \rightarrow \mathbb{R}$ and the control is $u(t) \in \mathbb{R}$.

Let us look at the Cauchy problem associated to (18)-(19), i.e., the following problem: Let $T>0, y^{0}$ : $(0, L) \rightarrow \mathbb{R}$ and $u:(0, T) \rightarrow \mathbb{R}$ be given, find $y:(0, T) \times(0, L) \rightarrow \mathbb{R}$ such that

$$
\begin{gathered}
y_{t}+y_{x}=0, t \in(0, T), x \in(0, L), \\
y(t, 0)=u(t), t \in(0, T), \\
y(0, x)=y^{0}(x), x \in(0, L) .
\end{gathered}
$$

One uses the following classical definition of a solution to the Cauchy problem (20)-(21)-(22).

Definition 8. Let $T>0, y^{0} \in L^{2}(0, L)$ and $u \in L^{2}(0, T)$. A solution to the Cauchy problem (20)-(21)-(22) is a function $y \in C^{0}\left([0, T] ; L^{2}(0, L)\right)$ such that, for every $\phi \in C^{1}([0, T] \times[0, L])$ such that

$$
\phi(t, L)=0, \forall t \in[0, T],
$$

one has, for every $\tau \in[0, T]$,

$$
-\int_{0}^{\tau} \int_{0}^{L}\left(\phi_{t}+\phi_{x}\right) y d x d t-\int_{0}^{\tau} u(t) \phi(t, 0) d t+\int_{0}^{L} y(\tau, x) \phi(\tau, x) d x-\int_{0}^{L} y^{0}(x) \phi(0, x) d x=0 .
$$

With this definition, one has the following standard result.

Theorem 9. Let $T>0, y^{0} \in L^{2}(0, L)$ and $u \in L^{2}(0, T)$. Then the Cauchy problem (20)-(21)-(22) has a unique solution. This solution satisfies

$$
\|y(\tau, \cdot)\|_{L^{2}(0, L)} \leqslant\left\|y^{0}\right\|_{L^{2}(0, L)}+\|u\|_{L^{2}(0, T)}, \forall \tau \in[0, T] .
$$


(See, e.g. [12, Section 3.1.1] for elementary proofs of this theorem). In fact, for this very simple control system, one can explicitly give the solution. Indeed, one easily checks that the function $y \in C^{0}\left([0, T] ; L^{2}(0, L)\right)$ defined by

$$
\begin{gathered}
y(t, x):=y^{0}(x-t), \forall(t, x) \in[0, T] \times(0, L) \text { such that } t \leqslant x, \\
y(t, x):=u(t-x), \forall(t, x) \in[0, T] \times(0, L) \text { such that } t>x,
\end{gathered}
$$

is a solution of the Cauchy problem (20)-(21)-(22).

Let us now study the controllability of the control system (18)-(19). We adopt the following definition.

Definition 10. Let $T>0$. The control system (18)-(19) is controllable in time $T$ if, for every $y^{0} \in L^{2}(0, L)$ and for every $y^{1} \in L^{2}(0, L)$, there exists $u \in L^{2}(0, T)$ such that the solution $y$ of the Cauchy problem (20)-(21)-(22) satisfies $y(T, \cdot)=y^{1}$.

If, in order to study this controllability, one tries the Lie bracket approach (which also works for linear finite-dimensional control systems: see, e.g., [12, Section 3.2]) one wants, for example, to understand what is $\left[f_{0}, f_{1}\right]$ for our control system (18)-(19). Inspired by (16), one considers the following control

$$
\begin{aligned}
& u_{\varepsilon}(t)=-1 \text { for } t \in(0, \varepsilon), \\
& u_{\varepsilon}(t)=1 \text { for } t \in(\varepsilon, 2 \varepsilon),
\end{aligned}
$$

where $\varepsilon \in(0, T / 2)$. We use this control and start from the state $y^{0}:=0$. Let $y^{\varepsilon}$ be the solution that we get for the Cauchy problem (20)-(21)-(22). Using (26) and (27), one gets, if $\varepsilon \in(0, L / 2]$,

$$
\begin{gathered}
y^{\varepsilon}(2 \varepsilon, x)=1, x \in(0, \varepsilon), \\
y^{\varepsilon}(2 \varepsilon, x)=-1, x \in(\varepsilon, 2 \varepsilon), \\
y^{\varepsilon}(2 \varepsilon, x)=0, x \in(2 \varepsilon, L) .
\end{gathered}
$$

Hence (compare with (16))

$$
\left\|\frac{y(2 \varepsilon, x)-y(0, x)}{\varepsilon^{2}}\right\|_{L^{2}(0, L)} \rightarrow+\infty \text { as } \varepsilon \rightarrow 0^{+} .
$$

Note that, for every $\phi$ in $C^{2}([0, L])$, one has, with

$$
\begin{gathered}
I_{\varepsilon}:=\int_{0}^{L} \phi(x)(y(2 \varepsilon, x)-y(0, x)) d x, \\
I_{\varepsilon}=-\left(\int_{\varepsilon^{\varepsilon}}^{2 \varepsilon} \phi(x) d x-\int_{0}^{\varepsilon} \phi(x) d x\right) \\
=-\int_{0}^{\varepsilon}(\phi(x+\varepsilon)-\phi(x)) d x \\
=-\int_{0}^{\varepsilon} \int_{x}^{x+\varepsilon} \phi^{\prime}(s) d s \\
=-\varepsilon^{2} \phi^{\prime}(0)+\int_{0}^{\varepsilon} \int_{x}^{x+\varepsilon}\left(\phi^{\prime}(0)-\phi^{\prime}(s)\right) d s .
\end{gathered}
$$

However, for every $s$ in $[0,2 \varepsilon]$,

$$
\left|\phi^{\prime}(s)-\phi^{\prime}(0)\right|=\left|\int_{0}^{s} \phi^{\prime \prime}(\tau) d \tau\right| \leqslant \sqrt{2 \varepsilon}\left\|\phi^{\prime \prime}\right\|_{L^{2}(0, L)} .
$$


Hence

$$
\frac{I_{\varepsilon}}{\varepsilon^{2}} \rightarrow-\phi^{\prime}(0) \text { as } \varepsilon \rightarrow 0^{+}
$$

In other words, as $\varepsilon \rightarrow 0^{+}$,

$$
\frac{y(2 \varepsilon, x)-y(0, x)}{\varepsilon^{2}} \rightarrow \delta_{0}^{\prime} \text { weakly in }\left(C^{2}([0, L])\right)^{\prime},
$$

where $\delta_{0}$ is the Dirac mass at 0 . So, we could say that, for our control system (18)-(19), $\left[f_{0}, f_{1}\right]=\delta_{0}^{\prime}$. Unfortunately, one does not know what to do with this $\delta_{0}^{\prime}$, which does not belong to the state space $L^{2}(0, L)$.

This example shows the difficulties to use iterated Lie brackets in infinite dimensions. However, for linear control systems in infinite dimension, one knows many methods to study the controllability. See, e.g., [12, Chaper $2]$ and using one of these methods one can prove

Theorem 11. The control system (18)-(19) is controllable in time $T$ if and only if $T \geqslant L$.

In fact one can give a simple explicit proof of Theorem 11. Let $y^{0} \in L^{2}(0, L)$ and $y^{1} \in L^{2}(0, L)$. Let us define $u \in L^{2}(0, T)$ by

$$
\begin{gathered}
u(t)=y^{1}(T-t), t \in(T-L, T), \\
u(t)=0, t \in(0, T-L) .
\end{gathered}
$$

Then, by (27), the solution $y$ of the Cauchy problem (20)-(21)-(22) satisfies

$$
y(T, x)=u(T-x)=y^{1}(x), x \in(0, L) .
$$

This shows that the control system (18)-(19) is controllable in time $T$.

The conclusion of this section is that

(1) Iterated Lie brackets do not work so well in infinite dimensions,

(2) One knows many methods to study the controllability of linear partial differential equations.

In particular the natural question is : What can be done for a partial differential control system having a linearized control system at the equilibrium which is not controllable? The goal of this talk is to present three methods (return method, quasi-static deformations, power series expansions) which are useful to handle this case.

\section{RETURN METHOD}

The return method has been introduced in [7] for a stabilization problem. It has been used for the first time in $[8,10]$ for the controllability of a partial differential equation, namely the Euler equations of incompressible fluids.

In order to explain this method, let us first consider the problem of local controllability of a control system in finite dimension. Thus we consider the control system

$$
\dot{x}=f(x, u),
$$

where $x \in \mathbb{R}^{n}$ is the state and $u \in \mathbb{R}^{m}$ is the control; we assume that $f$ is of class $C^{\infty}$ and satisfies

$$
f(0,0)=0 .
$$

The return method consists in reducing the local controllability of a nonlinear control system to the existence of suitable trajectories and to the controllability of linear systems. The idea is the following one: Assume that, 
for every positive real number $T$ and every positive real number $\varepsilon$, there exists a measurable bounded function $\bar{u}:[0, T] \rightarrow \mathbb{R}^{m}$ with $\|\bar{u}\|_{L^{\infty}(0, T)} \leqslant \varepsilon$ such that, if we denote by $\bar{x}$ the (maximal) solution of $\dot{\bar{x}}=f(\bar{x}, \bar{u}(t)$ ), $\bar{x}(0)=0$, then

$$
\bar{x}(T)=0,
$$

the linearized control system around $(\bar{x}, \bar{u})$ is controllable on $[0, T]$.

Then, from the inverse function theorem (see, e.g., [12, Theorem 3.6]), one gets the existence of $\eta>0$ such that, for every $x^{0} \in \mathbb{R}^{n}$ and for every $x^{1} \in \mathbb{R}^{n}$ satisfying

$$
\left|x^{0}\right|<\eta,\left|x^{1}\right|<\eta
$$

there exists $u \in L^{\infty}\left((0, T) ; \mathbb{R}^{m}\right)$ such that

$$
|u(t)-\bar{u}(t)| \leqslant \varepsilon, t \in[0, T]
$$

and such that, if $x:[0, T] \rightarrow \mathbb{R}^{n}$ is the solution of the Cauchy problem

$$
\dot{x}=f(x, u(t)), x(0)=x^{0},
$$

then

$$
x(T)=x^{1} .
$$

See Figure 1. Since $T>0$ and $\varepsilon>0$ are arbitrary, one gets that $\dot{x}=f(x, u)$ is small-time locally controllable at the equilibrium $(0,0) \in \mathbb{R}^{n} \times \mathbb{R}^{m}$. (For the definition of small-time local controllability, see Definition 1.)

Let us recall that the linearized control system around $(\bar{x}, \bar{u})$ is the time-varying control system

$$
\dot{y}=A(t) y+B(t) v,
$$

where the state is $y \in \mathbb{R}^{n}$, the control is $v \in \mathbb{R}^{m}$ and

$$
A(t):=\frac{\partial f}{\partial x}(\bar{x}(t), \bar{u}(t)), B(t):=\frac{\partial f}{\partial u}(\bar{x}(t), \bar{u}(t)) .
$$

For the linear control system (34), controllability on $[0, T]$ means that, for every $y_{0}$ and $y_{1}$ in $\mathbb{R}^{n}$, there exists a bounded measurable function $v:[0, T] \rightarrow \mathbb{R}^{m}$ such that, if $\dot{y}=A(t) y+B(t) v$ and $y(0)=y_{0}$, then $y(T)=y_{1}$.

Let us also recall (see, e.g., [12, Theorem 1.18]) that, if $A$ and $B$ are of class $C^{\infty}$, and if, for some $\bar{t} \in[0, T]$,

$$
\operatorname{Span}\left\{B_{i}(\bar{t}) u ; u \in \mathbb{R}^{m}, i \in \mathbb{N}\right\}=\mathbb{R}^{n},
$$

with $B_{i} \in C^{\infty}\left(\left[T_{0}, T_{1}\right] ; \mathcal{L}\left(\mathbb{R}^{m} ; \mathbb{R}^{n}\right)\right)$ defined by induction on $i$ in the following way

$$
B_{0}(t):=B(t), B_{i}(t):=\dot{B}_{i-1}(t)-A(t) B_{i-1}(t),
$$

then the linearized control system (34) is controllable on $[0, T]$.

Note that if one takes $\bar{u}=0$, then the above method is just Theorem 2. However, it may happen that (33) does not hold for $\bar{u}=0$, but holds for other choices of $\bar{u}$.

Remark 12. In fact, for many nonlinear partial differential equations, one does not use the linearized control system along the trajectory $(\bar{x}, \bar{u})$ to get the local controllability result along $(\bar{x}, \bar{u})$. One uses other adapted methods. These methods can rely on the controllability of some linear control systems which are not the linearized control system along the trajectory $(\bar{x}, \bar{u})$ (see [12, Remarks 4.11 and 6.30]). It can also rely on some specific methods which do not use the controllability of any linear control system. This last case appears in the papers [25] by Thierry Horsin and [23] by Olivier Glass. 


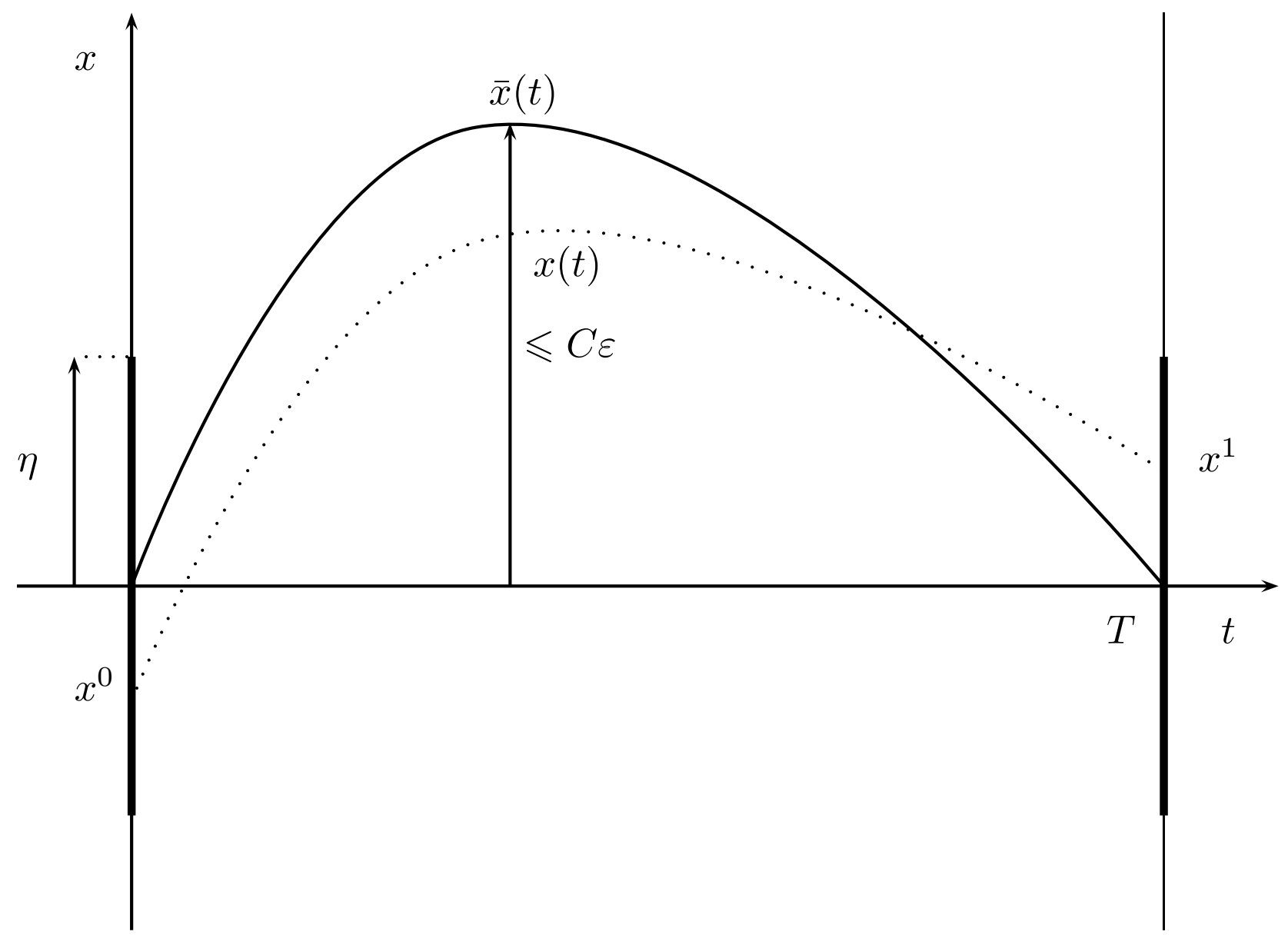

FiguRE 1. Return method

Let us show on simple examples in finite dimension how the return method can be used.

Example 13. We take $n=2, m=1$ and consider the control system

$$
\dot{x}_{1}=x_{2}^{3}, \dot{x}_{2}=u \text {. }
$$

For $\bar{u}=0$ and $\bar{x}=0$, the linearized control system around $(\bar{x}, \bar{u})$ is

$$
\dot{y}_{1}=0, \dot{y}_{2}=v
$$

which is clearly not controllable (one cannot change $y_{1}$ ). Let us now take $T>0$ and $\bar{u} \in C^{\infty}([0, T] ; \mathbb{R})$ such that

$$
\begin{gathered}
\int_{0}^{T / 2} \bar{u}(t) d t=0, \\
\bar{u}(T-t)=\bar{u}(t), \forall t \in[0, T] .
\end{gathered}
$$


Then one easily checks that

$$
\begin{gathered}
\bar{x}_{2}(T / 2)=0, \\
\bar{x}_{2}(T-t)=-\bar{x}_{2}(t), \forall t \in[0, T], \\
\bar{x}_{1}(T-t)=\bar{x}_{1}(t), \forall t \in[0, T] .
\end{gathered}
$$

In particular, we have

$$
\bar{x}_{1}(T)=0, \bar{x}_{2}(T)=0 .
$$

The linearized control system around $(\bar{x}, \bar{u})$ is

$$
\dot{y}_{1}=3 \bar{x}_{2}^{2}(t) y_{2}, \dot{y}_{2}=v .
$$

Hence

$$
A(t)=\left(\begin{array}{cc}
0 & 3 \bar{x}_{2}(t)^{2} \\
0 & 0
\end{array}\right), B(t)=\left(\begin{array}{l}
0 \\
1
\end{array}\right)
$$

and one easily sees that (35) holds if and only if

$$
\exists i \in \mathbb{N} \text { such that } \frac{d^{i} \bar{x}_{2}}{d t^{i}}(\bar{t}) \neq 0 .
$$

Note that (37) holds for at least a $\bar{t}$ in $[0, T]$ if (and only if) $\bar{u} \neq 0$. So (33) holds if (and only if) $\bar{u} \neq 0$. We recover that the control system (36) is small-time locally controllable at the equilibrium $(0,0) \in \mathbb{R}^{2} \times \mathbb{R}$.

Example 14. We return to the nonholonomic integrator (13) considered in Example 7: We take $n=3, m=2$ and the control system is

$$
\dot{x}_{1}=u_{1}, \dot{x}_{2}=u_{2}, \dot{x}_{3}=x_{1} u_{2}-x_{2} u_{1},
$$

where the state is $x=\left(x_{1}, x_{2}, x_{3}\right)^{\operatorname{tr}} \in \mathbb{R}^{3}$ and the control is $u=\left(u_{1}, u_{2}\right)^{\operatorname{tr}} \in \mathbb{R}^{2}$. Again one can check that the linearized control system around the trajectory $(\bar{x}, \bar{u})$ is controllable on $[0, T]$ if and only if $\bar{u} \neq 0$.

Note that, for the control system (38), it is easy to achieve the "return condition" (32). Indeed, let us impose that

$$
\bar{u}(T-t)=-\bar{u}(t), \forall t \in[0, T]
$$

Then

$$
\bar{x}(T-t)=\bar{x}(t), \forall t \in[0, T] .
$$

Indeed, let $y:[0, T] \rightarrow \mathbb{R}^{3}$ be defined by

$$
y(t)=\bar{x}(T-t), \forall t \in[0, T] .
$$

Then $y$, as $\bar{x}$, satisfies the ordinary differential equation

$$
\dot{x}_{1}=\bar{u}_{1}, \dot{x}_{2}=\bar{u}_{2}, \dot{x}_{3}=x_{1} \bar{u}_{2}-x_{2} \bar{u}_{1} .
$$

Hence, since $y$ and $\bar{x}$ are equal at the time $T / 2, y=\bar{x}$, which gives (40). From (40), we have

$$
\bar{x}(T)=\bar{x}(0)=0 .
$$

So the control system (38) is again small-time locally controllable at the equilibrium $(0,0) \in \mathbb{R}^{3} \times \mathbb{R}^{2}$ (a result which, as we have seen above, follows also directly from the Rashevski-Chow Theorem 6). 
The return method has been used to study the controllability of the following partial differential equations.

(1) Euler equations of incompressible fluids in [8,10], and, by Olivier Glass in [20,21],

(2) Navier-Stokes equations of incompressible fluids in [9, 14], and, by Andrei Fursikov et Oleg Imanuvilov, in $[19]$,

(3) Boussinesq equations, by Andrei Fursikov and Oleg Imanuvilov in [19],

(4) Burgers equation, by Thierry Horsin in [25],

(5) Saint-Venant equations in [11], a paper motivated by the prior paper [18] by François Dubois, Nicolas Petit and Pierre Rouchon (see also the next section),

(6) Vlasov-Poisson equations, by Olivier Glass in [22],

(7) 1-D Euler isentropic equations by Olivier Glass in [23],

(8) Schrödinger equations, by Karine Beauchard in [1] and in our joint work [4] with Karine Beauchard (these two papers are motivated by the prior paper [32] by Pierre Rouchon).

\section{QUASI-STATIC DEFORMATIONS}

We explain this method on a toy model related to the motion of a 1-D tank containing an inviscid incompressible irrotational fluid. The tank is subject to one-dimensional horizontal moves. We assume that the horizontal acceleration of the tank is small compared to the gravity constant and that the height of the fluid is small compared to the length of the tank. This motivates the use of the Saint-Venant equations [33] (also called shallow water equations) to describe the motion of the fluid; see e.g. [17, Sec. 4.2]. After suitable scaling arguments, the length of the tank and the gravity constant can be taken to be equal to 1; see [11]. Then the dynamics equations considered are -see [18] by François Dubois, Nicolas Petit and Pierre Rouchon-

$$
\begin{array}{r}
H_{t}(t, x)+(H v)_{x}(t, x)=0, \\
v_{t}(t, x)+\left(H+\frac{v^{2}}{2}\right)_{x}(t, x)=-u(t), \\
v(t, 0)=v(t, 1)=0, \\
\frac{\mathrm{d} s}{\mathrm{~d} t}(t)=u(t), \frac{\mathrm{d} D}{\mathrm{~d} t}(t)=s(t),
\end{array}
$$

where (see Figure 2)

- $H(t, x)$ is the height of the fluid at time $t$ and for $x \in[0,1]$,

- $v(t, x)$ is the horizontal water velocity of the fluid in a referential frame attached to the tank at time $t$ and for $x \in[0,1]$ (in the shallow water model, every points on the same vertical have the same horizontal velocity),

- $u$ is the horizontal acceleration of the tank in the absolute referential,

- $s$ is the horizontal velocity of the tank,

- $D$ is the horizontal displacement of the tank.

This is a control system, denoted $\Sigma$, where

- the state is $Y=(H, v, s, D)$,

- the control is $u \in \mathbb{R}$.

By scaling arguments again we may assume that, for every steady state, $H$, which is then a constant function, is equal to 1; see [11]. One is interested in the local controllability of the control system $\Sigma$ around the equilibrium point

$$
\left(Y_{e}, u_{e}\right):=((1,0,0,0), 0) .
$$

Of course, the total mass of the fluid is conserved so that, for every solution of (41) to (43), 


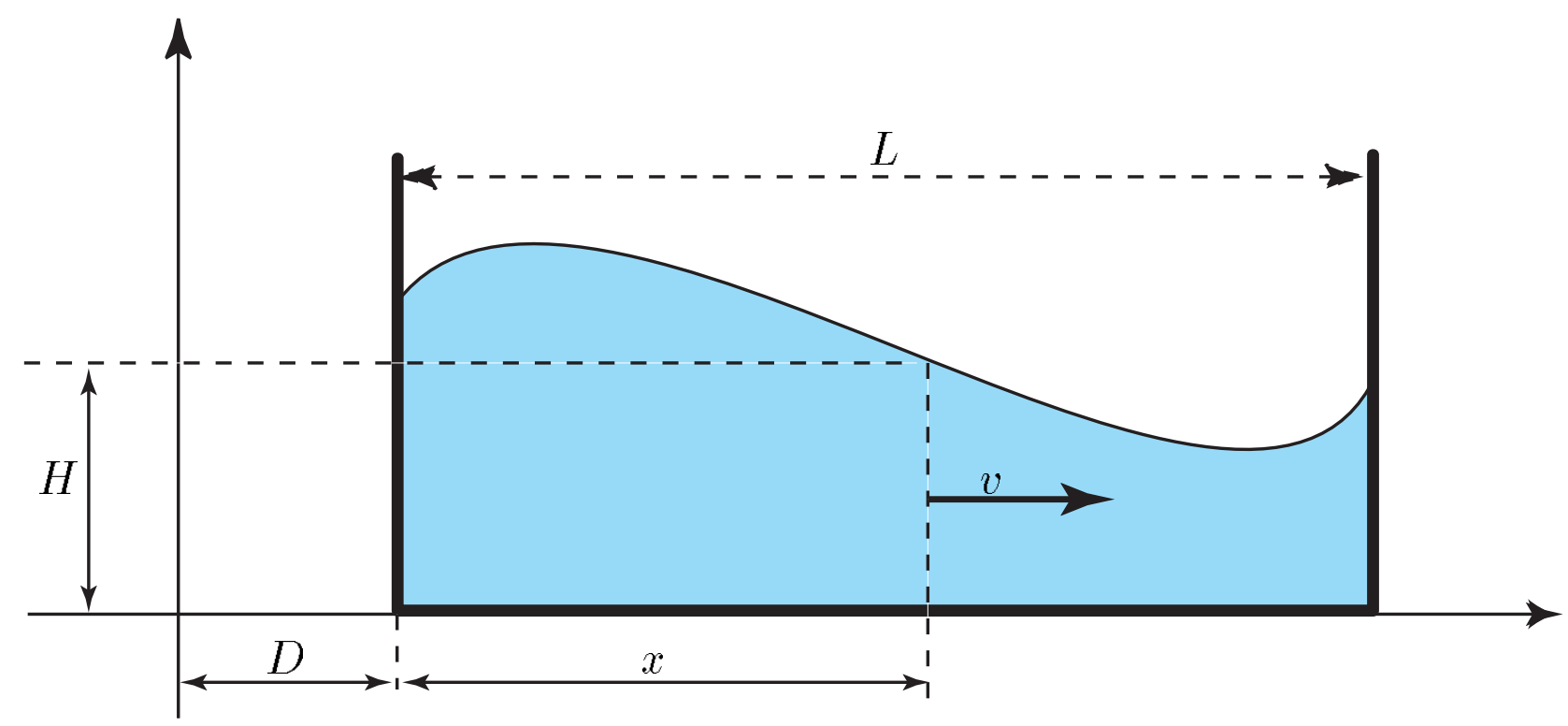

Figure 2. Fluid in the 1-D tank

$$
\frac{\mathrm{d}}{\mathrm{d} t} \int_{0}^{1} H(t, x) \mathrm{d} x=0
$$

(One gets (45) by integrating (41) on $[0,1]$ and by using (43) together with an integration by parts.) Moreover, if $H$ and $v$ are of class $C^{1}$, it follows from (42) and (43) that

$$
H_{x}(t, 0)=H_{x}(t, 1) \quad(=-u(t))
$$

Therefore we introduce the vector space $E$ of functions $Y=(H, v, s, D) \in C^{1}([0,1]) \times C^{1}([0,1]) \times \mathbb{R} \times \mathbb{R}$ such that

$$
H_{x}(0)=H_{x}(1), v(0)=v(1)=0,
$$

and consider the affine subspace $\mathcal{Y} \subset E$ of $Y=(H, v, s, D) \in E$ satisfying

$$
\int_{0}^{1} H(x) d x=1
$$

With these notations, we can define a trajectory of the control system $\Sigma$.

Definition of a trajectory. Let $T_{1}$ and $T_{2}$ be two real numbers satisfying $T_{1} \leqslant T_{2}$. A function $(Y, u)=$ $((H, v, s, D), u):\left[T_{1}, T_{2}\right] \rightarrow \mathcal{Y} \times \mathbb{R}$ is a trajectory of the control system $\Sigma$ if

(i) the functions $H$ and $v$ are of class $C^{1}$ on $\left[T_{1}, T_{2}\right] \times[0,1]$,

(ii) the functions $s$ and $D$ are of class $C^{1}$ on $\left[T_{1}, T_{2}\right]$ and the function $u$ is continuous on $[0, T]$,

(iii) the equations (41) to (44) hold for every $(t, x) \in\left[T_{1}, T_{2}\right] \times[0,1]$. 


\subsection{Controllability: results and open problems}

We are interested in the local controllability of $\Sigma$ around $\left(Y_{e}, u_{e}\right)$. Since one is looking for a local result, one first studies the linearized control system around the trajectory $\left(Y_{e}, u_{e}\right)$. Indeed, if this linearized control system is controllable, one can expect to get the local controllability of $\Sigma$ by using the inverse mapping theorem. (In fact, as mentionned in the introduction, for nonlinear partial differential control systems, this is not so direct when some "loss of derivatives" appear.) The linearized control system around $\left(Y_{e}, u_{e}\right)$ is

$$
\left(\Sigma_{\operatorname{lin}}\right)\left\{\begin{array}{l}
h_{t}+v_{x}=0, v_{t}+h_{x}=-u(t), v(t, 0)=v(t, 1)=0 \\
\frac{\mathrm{d} s}{\mathrm{~d} t}(t)=u(t), \frac{\mathrm{d} D}{\mathrm{~d} t}(t)=s(t)
\end{array}\right.
$$

where the state is $(h, v, s, D) \in \mathcal{Y}_{\text {lin }}$, with

$$
\mathcal{Y}_{\text {lin }}:=\left\{(h, v, s, D) \in E ; \int_{0}^{L} h d x=0\right\},
$$

and the control is $u \in \mathbb{R}$. It has been proved by F. Dubois, N. Petit and P. Rouchon in [18] that the linear control system (49) is not controllable (see also [29]). This noncontrollability property can be seen by noticing that (49) implies that, if

$$
h(0,1-x)=-h(0, x) \text { and } v(0,1-x)=v(0, x), \forall x \in[0,1],
$$

then

$$
h(t, 1-x)=-h(t, x) \text { and } v(t, 1-x)=v(t, x), \forall x \in[0,1], \forall t .
$$

Even if the control system (49) is not controllable, this control system, as it is proved in [18], is steady-state controllable, which means that one can move, from every steady state $\left(h_{0}, v_{0}, s_{0}, D_{0}\right):=\left(0,0,0, D_{0}\right)$ to every steady state $\left(h_{1}, v_{1}, s_{1}, D_{1}\right):=\left(0,0,0, D_{1}\right)$ for this control system (see also [29] when the tank has a non-straight bottom). This does not imply that the related property (move from $\left(1,0,0, D_{0}\right)$ to $\left(1,0,0, D_{1}\right)$ ) also holds for the nonlinear control system $\Sigma$ (even if $D_{0}$ and $D_{1}$ are small), as shown by the following example.

Example 15. Consider the two following control systems

$$
\begin{aligned}
& \dot{x}_{1}=x_{2}^{3}, \dot{x}_{2}=x_{1}+u, \dot{s}=u, \dot{D}=s, \\
& \dot{x}_{1}=x_{2}^{2}, \dot{x}_{2}=x_{1}+u, \dot{s}=u, \dot{D}=s,
\end{aligned}
$$

where, for both systems, the state is $x=\left(x_{1}, x_{2}, s, D\right) \in \mathbb{R}^{4}$ and the control is $u \in \mathbb{R}$. The set of steady states of these two control systems is the set of the $((0,0,0, a), 0)$ with $a \in \mathbb{R}$. These two control systems have the same linearized control system around $\left(x_{e}, u_{e}\right):=((0,0,0,0), 0)$, namely

$$
\dot{x}_{1}=0, \dot{x}_{2}=x_{1}+u, \dot{s}=u, \dot{D}=s .
$$

The linear control system (52) is not controllable, but this linear control is steady-state controllable: for (52), one can move from $\left(0,0,0, a_{1}\right)$ to $\left(0,0,0, a_{2}\right)$ whatever are $a_{1} \in \mathbb{R}$ and $a_{2} \in \mathbb{R}$. But

- For control system (50), one can move from $\left(0,0,0, a_{1}\right)$ to $\left(0,0,0, a_{2}\right)$ whatever are $a_{1} \in \mathbb{R}$ and $a_{2} \in \mathbb{R}$

- For control system (51), one can never move from $\left(0,0,0, a_{1}\right)$ to $\left(\left(0,0,0, a_{2}\right)\right.$ whatever are $a_{1} \in \mathbb{R}$ and $a_{2} \in \mathbb{R}$ with $a_{1} \neq a_{2}$.

Let us introduce our definition of local controllability $\mathrm{C}(T)$ and our definition of steady-state local controllability $\mathrm{S}(T)$. For $w \in C^{1}([0, L])$, let

$$
|w|_{1}:=\operatorname{Max}\left\{|w(x)|+\left|w_{x}(x)\right| ; x \in[0, L]\right\} .
$$


The definition of $\mathrm{C}(T)$ is the following one.

Definition of $\mathrm{C}(T)$. Let $T>0$. The control system $\Sigma$ satisfies the property $\mathrm{C}(T)$ if, for every $\epsilon$, there exists $\eta>0$ such that, for every $Y_{0}=\left(H_{0}, v_{0}, s_{0}, D_{0}\right) \in \mathcal{Y}$, and for every $Y_{1}=\left(H_{1}, v_{1}, s_{1}, D_{1}\right) \in \mathcal{Y}$ such that

$$
\left|H_{0}-1\right|_{1}+\left|v_{0}\right|_{1}+\left|H_{1}-1\right|_{1}+\left|v_{1}\right|_{1}+\left|s_{0}\right|+\left|s_{1}\right|+\left|D_{0}\right|+\left|D_{1}\right|<\eta
$$

there exists a trajectory

$$
(Y, u):[0, T] \rightarrow \mathcal{Y} \times \mathbb{R}, t \mapsto((H(t), v(t), s(t), D(t)), u(t))
$$

of the control system $\Sigma$ such that

$$
Y(0)=Y_{0} \text { and } Y(T)=Y_{1}
$$

and, for every $t \in[0, T]$,

$$
|H(t)-1|_{1}+|v(t)|_{1}+|s(t)|+|D(t)|+|u(t)|<\epsilon .
$$

The definition of our steady-state local controllability property $\mathrm{S}(T)$ is the following one.

Definition of $\mathrm{S}(T)$. Let $T>0$. The control system $\Sigma$ satisfies the property $\mathrm{S}(T)$ if, for every $\epsilon$, there exists $\eta>0$ such that, for every $D_{0} \in \mathbb{R}$, and for every $D_{1} \in \mathbb{R}$ such that

$$
\left|D_{0}\right|+\left|D_{1}\right|<\eta
$$

there exists a trajectory

$$
(Y, u):[0, T] \rightarrow \mathcal{Y} \times \mathbb{R}, t \mapsto((H(t), v(t), s(t), D(t)), u(t))
$$

of the control system $\Sigma$ such that

$$
Y(0)=\left(0,0,0, D_{0}\right) \text { and } Y(T)=\left(0,0,0, D_{1}\right),
$$

and, for every $t \in[0, T]$,

$$
|H(t)-1|_{1}+|v(t)|_{1}+|s(t)|+|D(t)|+|u(t)|<\epsilon .
$$

Clearly $\mathrm{C}(T)$ implies $\mathrm{S}(T)$, and, for $T \leq T^{\prime}, \mathrm{C}(T)$ implies $\mathrm{C}\left(T^{\prime}\right)$ and $\mathrm{S}(T)$ implies $\mathrm{S}\left(T^{\prime}\right)$. Using the characteristics of the hyperbolic system (41)-(42) one easily sees that $\mathrm{S}(T)$ does not hold if $T<1$. The following theorem is proved in [11].

Theorem 16. Property $\mathrm{C}(T)$ holds for $T$ large enough. $5.2)$.

The proof of Theorem 16 given in [11] relies on the return method and quasi-static deformations (see Section One has the following open problems.

Open Problem 17. What is the value of

$$
T_{c}:=\inf \{T>0 ; \mathrm{C}(T) \text { holds }\} .
$$

Open Problem 18. What is the value of

$$
T_{s}:=\inf \{T>0 ; \mathrm{S}(T) \text { holds }\} .
$$


Our guess is that

$$
T_{c}=T_{s} \geqslant 2 .
$$

Note that, for the linearized control system (49), it is proved that the steady-state controllability holds for every $T>1$. So, if our guess (58) holds, the nonlinearity which has helped us in order to get the local controllability, would impose an extra time for the steady-state local controllability.

\subsection{A toy model}

In this section, we give the main ideas (in particular the quasi-static deformation method) of the proof of Theorem 16 on a very simple finite dimensional control system which shares some properties with our control system $\Sigma$. This will be our toy model, denoted by $\mathcal{T}$. We also propose on this toy model a method which could be tried on $\Sigma$ in order to get an upper bound on $T_{c}$.

For a function $w:[0,1] \rightarrow \mathbb{R}$, we denote by $w^{\mathrm{ev}}$ "the even part" of $w$ and by $w^{\text {od }}$ the odd part of $w$ :

$$
w^{\mathrm{ev}}(x):=\frac{1}{2}(w(x)+w(1-x)), w^{\mathrm{od}}(x):=\frac{1}{2}(w(x)-w(1-x)) .
$$

Then, if $h:=1-H$, one has from (41) to (44)

$$
\begin{aligned}
& \left\{\begin{array}{l}
h_{t}^{\mathrm{od}}+v_{x}^{\mathrm{ev}}=-\left(h^{\mathrm{ev}} v^{\mathrm{ev}}+h^{\mathrm{od}} v^{\mathrm{od}}\right)_{x}, v_{t}^{\mathrm{ev}}+h_{x}^{\mathrm{od}}=-u(t)-\left(v^{\mathrm{ev}} v^{\mathrm{od}}\right)_{x}, \\
v^{\mathrm{ev}}(t, 0)=v^{\mathrm{ev}}(t, 1)=0, \frac{\mathrm{d} s}{\mathrm{~d} t}(t)=u(t), \frac{\mathrm{d} D}{\mathrm{~d} t}(t)=s(t)
\end{array}\right. \\
& \left\{\begin{array}{l}
h_{t}^{\mathrm{ev}}+v_{x}^{\text {od }}=-\left(h^{\mathrm{ev}} v^{\text {od }}+h^{\text {od }} v^{\mathrm{ev}}\right)_{x}, v_{t}^{\text {od }}(t, x)+h_{x}^{\mathrm{ev}}=-\frac{1}{2}\left(\left(v^{\mathrm{ev}}\right)^{2}+\left(v^{\text {od }}\right)^{2}\right)_{x} \\
v^{\text {od }}(t, 0)=v^{\text {od }}(t, 1)=0
\end{array}\right.
\end{aligned}
$$

together with the initial conditions

$$
\begin{gathered}
h^{\text {od }}(0, x)=h_{0}^{\text {od }}(x), v^{\text {ev }}(0, x)=v_{0}^{\text {ev }}(x), s_{1}(0)=s_{1}, D_{1}(0)=D_{1}, \\
h^{\text {ev }}(0, x)=h_{0}^{\text {ev }}(x), v^{\text {od }}(0, x)=v_{0}^{\text {od }}(x) .
\end{gathered}
$$

The linearized control system of the part (59) is

$$
\left\{\begin{array}{l}
h_{t}^{\text {od }}+v_{x}^{\text {ev }}=0, v_{t}^{\text {ev }}+h_{x}^{\text {od }}=-u(t), v^{\mathrm{ev}}(t, 0)=v^{\mathrm{ev}}(t, 1)=0 \\
\frac{\mathrm{d} s}{\mathrm{~d} t}(t)=u(t), \frac{\mathrm{d} D}{\mathrm{~d} t}(t)=s(t)
\end{array}\right.
$$

Let us consider $(63)$ as a control system where the control is $u$ and where the state $\left(h^{\text {od }}, v^{\text {ev }}, s, D\right) \in C^{1}([0,1]) \times$ $C^{1}([0,1]) \times \mathbb{R} \times \mathbb{R}$ satisfies

$$
h^{\mathrm{od}}(1-x)=-h^{\mathrm{od}}(x), v^{\mathrm{ev}}(L-x)=v^{\mathrm{ev}}(x), v^{\mathrm{ev}}(0)=v^{\mathrm{ev}}(L)=0 .
$$

Note that, by [18], this control system is controllable (in every time $T>1$ ). When one linearizes the first two equations of (59) and the two equations (60), one gets the usual wave equations. A natural analogue of the wave equation in finite dimension is the oscillator equation. Hence a natural analogous to our control system (59)-(60) is

$$
(\mathcal{T}) \dot{x}_{1}=x_{2}, \dot{x}_{2}=-x_{1}+x_{2} x_{3}+u, \dot{x}_{3}=x_{4}, \dot{x}_{4}=-x_{3}+2 x_{1} x_{2}, \dot{s}=u, \dot{D}=s
$$

where the state is $x=\left(x_{1}, x_{2}, x_{3}, x_{4}, s, D\right) \in \mathbb{R}^{6}$ and the control is $u \in \mathbb{R}$. (The quadratic terms in (64) need some specific properties but could be much more general than the one chosen here.) This control system is our toy model. 
The linearized control system of $\mathcal{T}$ around $(0,0) \in \mathbb{R}^{6} \times \mathbb{R}$ is

$$
\dot{x}_{1}=x_{2}, \dot{x}_{2}=-x_{1}+u, \dot{x}_{3}=x_{4}, \dot{x}_{4}=-x_{3}, \dot{s}=u, \dot{D}=s .
$$

This linear control system is not controllable (look at $\left(x_{3}, x_{4}\right)$ ). But, as one easily checks, (65) is steady-state controllable for arbitrary time $T$, that is, for every $\left(D_{0}, D_{1}\right) \in \mathbb{R}^{2}$ and for every $T>0$, there exists a trajectory $\left(\left(x_{1}, x_{2}, x_{3}, x_{4}, s, D\right), u\right):[0, T] \rightarrow \mathbb{R}^{6} \times \mathbb{R}$ of the linear control system (65) such that

$$
\begin{gathered}
x_{1}(0)=x_{2}(0)=x_{3}(0)=x_{4}(0)=0, s(0)=0, D(0)=D_{0}, \\
x_{1}(T)=x_{2}(T)=x_{3}(T)=x_{4}(T)=0, s(T)=0, D(T)=D_{1}
\end{gathered}
$$

But the same does not hold for the nonlinear control system $\mathcal{T}$ : one can check that one needs $T>2 \pi$ in order to have the steady-state local controllability for the nonlinear control system $\mathcal{T}$.

Let us now prove the following large time local controllability of $\mathcal{T}$.

Proposition 19. There exists $T>0$ and $\delta>0$ such that, for every $a \in \mathbb{R}^{6}$ and every $b \in \mathbb{R}^{6}$ with $|a|<\delta$ and $|b|<\delta$, there exists $u \in L^{\infty}(0, T)$ such that, if $x=\left(x_{1}, x_{2}, x_{3}, x_{4}, s, D\right):[0, T] \rightarrow \mathbb{R}^{6}$ is the solution of the Cauchy problem

$$
\dot{x}_{1}=x_{2}, \dot{x}_{2}=-x_{1}+x_{2} x_{3}+u, \dot{x}_{3}=x_{4}, \dot{x}_{4}=-x_{3}+2 x_{1} x_{2}, \dot{s}=u, \dot{D}=s, x(0)=a,
$$

then $x(T)=b$.

Let us prove this proposition by using the return method and quasi-static deformations. In order to use the return method one needs, at least, to know trajectories of the control system $\mathcal{T}$ such that the linearized control systems around these trajectories are controllable. The simplest trajectories one can consider are the trajectories

$$
\left(\left(x_{1}^{\gamma}, x_{2}^{\gamma}, x_{3}^{\gamma}, x_{4}^{\gamma}, s^{\gamma}, D^{\gamma}\right), u^{\gamma}\right)=\left(\left(\gamma, 0,0,0, \gamma t, \gamma t^{2} / 2\right), \gamma\right),
$$

where $\tau_{1}>0$ is fixed, $\gamma$ is any real number different from 0 and $t \in\left[0, \tau_{1}\right]$. The linearized control system around the trajectory $\left(x^{\gamma}, u^{\gamma}\right):=\left(\left(x_{1}^{\gamma}, x_{2}^{\gamma}, x_{3}^{\gamma}, x_{4}^{\gamma}, s^{\gamma}, D^{\gamma}\right), u^{\gamma}\right)$ is the linear control system

$$
\dot{x}_{1}=x_{2}, \dot{x}_{2}=-x_{1}+u, \dot{x}_{3}=x_{4}, \dot{x}_{4}=-x_{3}+2 \gamma x_{2}, \dot{s}=u, \dot{D}=s .
$$

Using the usual Kalman rank condition for controllability (Theorem 3), one easily checks that this linear control system is controllable if and only if $\gamma \neq 0$. Let us now choose $\gamma \neq 0$. Then, since the linearized control system around $\left(x^{\gamma}, u^{\gamma}\right)$ is controllable, there exists $\delta_{1}>0$ such that for every $a \in B\left(x^{\gamma}(0), \delta_{1}\right):=\left\{x \in \mathbb{R}^{6} ;\left|x-x^{\gamma}(0)\right|<\right.$ $\left.\delta_{1}\right\}$ and for every $b$ in $B\left(x^{\gamma}\left(\tau_{1}\right), \delta_{1}\right):=\left\{x \in \mathbb{R}^{6} ;\left|x-x^{\gamma}\left(\tau_{1}\right)\right|<\delta_{1}\right\}$ there exists $u \in L^{\infty}\left(\left[0, \tau_{1}\right] ; \mathbb{R}\right)$ such that

$$
\left(\dot{x}_{1}=x_{2}, \dot{x}_{2}=-x_{1}+x_{2} x_{3}+u, \dot{x}_{3}=x_{4}, \dot{x}_{4}=-x_{3}+2 x_{1} x_{2}, \dot{s}=D, \dot{D}=u, x(0)=a\right) \Rightarrow\left(x\left(T_{0}\right)=b\right) .
$$

Hence, by the continuity of the solutions of the Cauchy problem with respect to the initial condition, in order to prove Proposition 19 it suffices to check that

(i) there exist $\tau_{2}>0$ and a trajectory $(\tilde{x}, \tilde{u}):\left[0, \tau_{2}\right] \rightarrow \mathbb{R}^{6} \times \mathbb{R}$ of the control system $\mathcal{T}$ such that $\tilde{x}(0)=0$ and $\left|\tilde{x}\left(\tau_{2}\right)-x^{\gamma}(0)\right|<\delta_{1}$.

(ii) there exist $\tau_{3}>0$ and a trajectory $(\hat{x}, \hat{u}):\left[0, \tau_{3}\right] \rightarrow \mathbb{R}^{6} \times \mathbb{R}$ of the control system $\mathcal{T}$ such that $\hat{x}\left(\tau_{3}\right)=0$ and $\left|\hat{x}(0)-x^{\gamma}\left(\tau_{1}\right)\right|<\delta_{1}$.

In order to prove (i) we consider quasi-static deformations. Let $g \in C^{2}([0,1] ; \mathbb{R})$ be such that

$$
g(0)=0, g(1)=1
$$


Let $\tilde{u}:[0,1 / \epsilon] \rightarrow \mathbb{R}$ be defined by $\tilde{u}(t)=\gamma g(\epsilon t)$. Let $\tilde{x}:=\left(\tilde{x}_{1}, \tilde{x}_{2}, \tilde{x}_{3}, \tilde{x}_{4}, \tilde{s}, \tilde{D}\right):[0,1 / \epsilon] \rightarrow \mathbb{R}^{6}$ be defined by

$$
\dot{\tilde{x}}_{1}=\tilde{x}_{2}, \dot{\tilde{x}}_{2}=-\tilde{x}_{1}+\tilde{x}_{2} \tilde{x}_{3}+\tilde{u}, \dot{\tilde{x}}_{3}=\tilde{x}_{4}, \dot{\tilde{x}}_{4}=-\tilde{x}_{3}+2 \tilde{x}_{1} \tilde{x}_{2}, \dot{\tilde{s}}=\tilde{u}, \dot{\tilde{D}}=\tilde{s}, \tilde{x}(0)=0 .
$$

One easily checks that

$$
\tilde{x}(1 / \epsilon) \rightarrow(\gamma, 0,0,0,0,0) \text { as } \epsilon \rightarrow 0,
$$

which ends the proof of (i).

In order to get (ii) one needs just to modify a little bit the above construction.

Remark 20. The linearized system of

$$
\dot{x}_{1}=x_{2}, \dot{x}_{2}=-x_{1}+x_{2} x_{3}+\gamma, \dot{x}_{3}=x_{4}, \dot{x}_{4}=-x_{3}+2 x_{1} x_{2},
$$

at the equilibrium $(\gamma, 0,0,0)$ has $i$ and $-i$ for eigenvalues. This is why the quasi-static deformation is so easy to perform. If this linearized system would have eigenvalues with strictly positive real part, it is still possible to perform in some cases the quasi-static deformation by stabilizing the equilibriums by suitable feedbacks as it has been pointed out in [15] for the first time for a partial differential equation.

Quasi-static deformations have been used for the following partial differential equations.

(1) Saint-Venant equations (also called shallow water equations) in [11],

(2) Semilinear heat equations in [15], a joint work with Emmanuel Trélat,

(3) Navier-Stokes equations for incompressible fluids in [34] by Michael Schmidt and Emmanuel Trélat,

(4) Schrödinger equations in [1] by Karine Beauchard, and in our joint work [4] with her,

(5) Semilinear wave equations in [16], a joint work with Emmanuel trélat.

\section{POWER SERIES EXPANSION}

The method which we have used in order to prove Proposition 19 has an important drawback: Due to the quasi-static deformation parts, it leads to estimates on the time $T$ for controllability which are too conservative. Let us now propose another method which gives a good estimate on the time for local controllability. This method is classical in finite dimension -see for example [26] and the references therein- and has been used for the first time in infinite dimension for a KdV control system in [13]. It consists in looking for "higher order variations" which allows us to move in the directions which are missed by the controllability of the linearized control system. These directions are $\pm(0,0,1,0,0,0)$ and $\pm(0,0,0,1,0,0)$ for our toy control system $\mathcal{T}$. For the control system $\Sigma$, these directions are given by $(h, v, 0,0) \in \mathcal{Y}_{\text {lin }}$, with $h(1-x)=h(x)$ and $v(1-x)=-v(x)$. Let us describe this method on $\mathcal{T}$ in order to get that Proposition 19 holds for every $T>\pi$.

One first looks to the linearized control system around 0, i.e. the linear control system (65). Let $T>0$ and let $\left(e_{i}\right)_{i \in\{1, \ldots, 6\}}$ be the usual basis of $\mathbb{R}^{6}$. One easily sees that, for every $i \in \mathcal{I}_{c}:=\{1,2,5,6\}$, there exists $u_{i} \in L^{\infty}(0, T)$ such that

$$
\left(\dot{x}_{1}=x_{2}, \dot{x}_{2}=-x_{1}+u_{i}, \dot{x}_{3}=x_{4}, \dot{x}_{4}=-x_{3}, \dot{s}=u_{i}, \dot{D}=s, x(0)=0\right) \Rightarrow\left(x(T)=e_{i}\right) .
$$

Let us assume for the moment that, for every $i \in \mathcal{I}_{u}:=\{3,4\}$, there exist $u_{i}^{ \pm} \in L^{\infty}(0, T)$ such that

$$
\left(\dot{x}_{1}=x_{2}, \dot{x}_{2}=-x_{1}+u_{i}^{ \pm}, \dot{x}_{3}=x_{4}, \dot{x}_{4}=-x_{3}+2 x_{1} x_{2}, \dot{s}=u_{i}^{ \pm}, \dot{D}=s, x(0)=0\right) \Rightarrow\left(x(T)= \pm e_{i}\right) .
$$

Note that in the left hand side of (69), we have put $\dot{x}_{2}=-x_{1}+u_{i}^{ \pm}$and not $\dot{x}_{2}=-x_{1}+x_{2} x_{3}+u_{i}^{ \pm}$. The reason is that the $x_{i}$ with $i \in \mathcal{I}_{c}$ and $u$ are considered of order 1 , and the $x_{i}$ with $i \in \mathcal{I}_{u}$ are considered to be of order 
2. With this choice of scaling, the left hand side of (69) is the approximation of order 2 of the control system

$\mathcal{T}$. Then, let $b:=\sum_{i=1}^{6} b_{i} e_{i}$. Let, for $i \in \mathcal{I}_{u}$,

$$
\text { if } b_{i} \geq 0, u_{i}:=u_{i}^{+} \text {and if } b_{i}<0, u_{i}:=u_{i}^{-} \text {. }
$$

Let $u \in L^{\infty}(0, T)$ be defined by

$$
u:=\sum_{i \in \mathcal{I}_{c}} b_{i} u_{i}+\sum_{i \in \mathcal{I}_{u}}\left|b_{i}\right|^{1 / 2} u_{i} .
$$

Let $x:[0, T] \rightarrow \mathbb{R}^{6}$ be the solution of the Cauchy problem

$$
\dot{x}_{1}=x_{2}, \dot{x}_{2}=-x_{1}+x_{2} x_{3}+u, \dot{x}_{3}=x_{4}, \dot{x}_{4}=-x_{3}+2 x_{1} x_{2}, \dot{s}=u, \dot{D}=s, x(0)=0 .
$$

Then straightforward estimates lead to

$$
x(T)=b+o(b) \text { as } b \rightarrow 0
$$

Hence, using the Brouwer fixed point theorem (and standard estimates on ordinary differential equations), one gets the local controllability of $\mathcal{T}$ (around 0 ) in time $T$ (and therefore Proposition 19). It remains to prove the existence of $u_{i}^{ \pm} \in L^{\infty}(0, T)$ for every $i \in \mathcal{I}_{u}:=\{3,4\}$. Easy computations show that, if

$$
\dot{x}_{1}=x_{2}, \dot{x}_{3}=x_{4}, \dot{x}_{4}=-x_{3}+2 x_{1} x_{2},
$$

then

$$
x_{3}(T)=\int_{0}^{T} x_{1}^{2}(t) \cos (T-t) d t, x_{4}(T)=x_{1}^{2}(T)-\int_{0}^{T} x_{1}^{2}(t) \sin (T-t) d t .
$$

From (70), it is not hard to get that the existence of $u_{i}^{ \pm} \in L^{\infty}(0, T)$ for every $i \in \mathcal{I}_{u}$ holds if (and only if) $T>\pi$.

This power series expansion method has been used for the following partial differential equations.

(1) Korteweg-de Vries equations in [13], a joint work with Emmanuelle Crépeau, and in [5] by Eduardo Cerpa. These two works are motivated by the prior work [31] by Lionel Rosier.

(2) Schrödinger equations in [4], a joint work with Karine Beauchard, already mentioned above.

\section{REFERENCES}

[1] Karine Beauchard. Local controllability of a 1-D Schrödinger equation. J. Math. Pures Appl. (9), 84(7):851-956, 2005.

[2] Karine Beauchard. Local controllability of a 1D beam equation. Preprint, CMLA, Submitted for publication, 2005.

[3] Karine Beauchard. Controllability of a quantum particle in a 1D infinite square potential with variable length. Preprint, CMLA, Submitted for publication, 2006.

[4] Karine Beauchard and Jean-Michel Coron. Controllability of a quantum particle in a moving potential well. J. Functional Analysis, 232:328-389, 2006.

[5] Eduardo Cerpa. Exact controllability of a nonlinear Korteweg-de Vries equation on a critical spatial domain. submitted to SIAM J. Control Optim., 2006

[6] Wei-Liang Chow. Über Systeme von linearen partiellen Differentialgleichungen erster Ordnung. Math. Ann., 117:98-105, 1939.

[7] Jean-Michel Coron. Global asymptotic stabilization for controllable systems without drift. Math. Control Signals Systems, $5(3): 295-312,1992$.

[8] Jean-Michel Coron. Contrôlabilité exacte frontière de l'équation d'Euler des fluides parfaits incompressibles bidimensionnels. C. R. Acad. Sci. Paris Sér. I Math., 317(3):271-276, 1993.

[9] Jean-Michel Coron. On the controllability of the 2-D incompressible Navier-Stokes equations with the Navier slip boundary conditions. ESAIM Control Optim. Calc. Var., 1:35-75 (electronic), 1995/96.

[10] Jean-Michel Coron. On the controllability of 2-D incompressible perfect fluids. J. Math. Pures Appl. (9), 75(2):155-188, 1996. 
[11] Jean-Michel Coron. Local controllability of a 1-D tank containing a fluid modeled by the shallow water equations. ESAIM Control Optim. Calc. Var., 8:513-554, 2002. A tribute to J. L. Lions.

[12] Jean-Michel Coron. Control and nonlinearity. Mathematical Surveys and Monographs. American Mathematical Society, Providence, RI, to appear.

[13] Jean-Michel Coron and Emmanuelle Crépeau. Exact boundary controllability of a nonlinear KdV equation with critical lengths. J. Eur. Math. Soc. (JEMS), 6(3):367-398, 2004.

[14] Jean-Michel Coron and Andrei V. Fursikov. Global exact controllability of the 2D Navier-Stokes equations on a manifold without boundary. Russian J. Math. Phys., 4(4):429-448, 1996.

[15] Jean-Michel Coron and Emmanuel Trélat. Global steady-state controllability of one-dimensional semilinear heat equations. SIAM J. Control Optim., 43(2):549-569 (electronic), 2004.

[16] Jean-Michel Coron and Emmanuel Trélat. Global steady-state stabilization and controllability of 1D semilinear wave equations. Commun. Contemp. Math., 8(4):535-567, 2006.

[17] Lokenath Debnath. Nonlinear water waves. Academic Press Inc., Boston, MA, 1994.

[18] François Dubois, Nicolas Petit, and Pierre Rouchon. Motion planning and nonlinear simulations for a tank containing a fluid. ECC, 1999.

[19] Andrei V. Fursikov and Oleg Yu. Imanuvilov. Exact controllability of the Navier-Stokes and Boussinesq equations. Russian Math. Surveys, 54:565-618, 1999.

[20] Olivier Glass. Contrôlabilité exacte frontière de l'équation d'Euler des fluides parfaits incompressibles en dimension $3 . C$. $R$. Acad. Sci. Paris Sér. I Math., 325(9):987-992, 1997.

[21] Olivier Glass. Exact boundary controllability of 3-D Euler equation. ESAIM Control Optim. Calc. Var., 5:1-44 (electronic), 2000.

[22] Olivier Glass. On the controllability of the Vlasov-Poisson system. J. Differential Equations, 195(2):332-379, 2003.

[23] Olivier Glass. On the controllabilty of the 1-D isentropic Euler equation. accepted for publication in J. Eur. Math. Soc. (JEMS), 2006.

[24] Robert Hermann. On the accessibility problem in control theory. In Internat. Sympos. Nonlinear Differential Equations and Nonlinear Mechanics, pages 325-332. Academic Press, New York, 1963.

[25] Thierry Horsin. On the controllability of the Burgers equation. ESAIM Control Optim. Calc. Var., 3:83-95 (electronic), 1998.

[26] Matthias Kawski. High-order small-time local controllability. In H.J. Sussmann, editor, Nonlinear controllability and optimal control, volume 133 of Monogr. Textbooks Pure Appl. Math., pages 431-467. Dekker, New York, 1990.

[27] Claude Lobry. Contrôlabilité des systèmes non linéaires. SIAM J. Control, 8:573-605, 1970.

[28] Tadashi Nagano. Linear differential systems with singularities and an application to transitive Lie algebras. J. Math. Soc. Japan, 18:398-404, 1966.

[29] Nicolas Petit and Pierre Rouchon. Dynamics and solutions to some control problems for water-tank systems. IEEE Trans. Automat. Control, 47(4):594-609, 2002.

[30] Petr K. Rashevski. About connecting two points of complete nonholonomic space by admissible curve. Uch Zapiski ped. inst. Libknexta, 2:83-94, 1938.

[31] Lionel Rosier. Exact boundary controllability for the Korteweg-de Vries equation on a bounded domain. ESAIM Control Optim. Calc. Var., 2:33-55 (electronic), 1997.

[32] Pierre Rouchon. Control of a quantum particle in a moving potential well. In Lagrangian and Hamiltonian methods for nonlinear control 2003, pages 287-290. IFAC, Laxenburg, 2003.

[33] Adhémar Jean Claude Barré de Saint-Venant. Théorie du mouvement non permanent des eaux, avec applications aux crues des rivières et à l'introduction des marées dans leur lit. C. R. Acad. Sci. Paris Sér. I Math., 53:147-154, 1871.

[34] Michael Schmidt and Emmanuel Trélat. Controllability of Couette flows. Commun. Pure Appl. Anal., 5(1):201-211, 2006. 